\title{
World Journal of Pediatric Surgery \\ Laparoscopic surgery for congenital biliary dilatation accompanied by accessory hepatic ducts: a report of seven cases
}

Yuebin Zhang (D) $,{ }^{1} \mathrm{Jia} \mathrm{Yu},{ }^{2}$ Chen Qingjiang, ${ }^{1}$ Duote Cai, ${ }^{1}$ Zhigang Gao ${ }^{1}$

To cite: Zhang Y, Yu J, Qingjiang C, et al. Laparoscopic surgery for congenital biliary dilatation accompanied by accessory hepatic ducts: a report of seven cases. World JnI Ped Surgery 2022;5:e000359. doi:10.1136/wjps-2021-000359

Received 20 September 2021 Accepted 6 December 2021
Check for updates

(C) Author(s) (or their employer(s)) 2022. Re-use permitted under CC BY-NC. No commercial re-use. See rights and permissions. Published by BMJ.

${ }^{1}$ General Pediatric Surgery, Zhejiang University School of Medicine Children's Hospital, Hangzhou, Zhejiang, China PICU, Zhejiang University School of Medicine Children's Hospital, Hangzhou, Zhejiang, China

Correspondence to Dr Zhigang Gao; ebwk@zju. edu.cn
Congenital biliary dilatation (CBD) is a congenital biliary tract anomaly characterized by expansion of the common bile duct and is thus known clinically as a choledochal cyst. The form of CBD varies, and the commonly used clinical classification was described by Todani et al. ${ }^{1}$ Pancreaticobiliary maljunction is reported to be present in 30\%-96\% of patients with CBD. ${ }^{2} 3$ The main aims of laparoscopic surgery include resection of the dilated biliary ducts, gallbladder, pancreaticbile diversion, and biliary tract reconstruction. ${ }^{4}$ Accessory hepatic ducts (AHDs) are extrasegmental bile ducts that often drain a part of the liver and can join the biliary system at any position, such as the common bile duct, common hepatic duct (CHD), right hepatic duct or the gallbladder. AHDs are seldom seen on preoperative imaging, but they increase the difficulty of surgery and the risk of postoperative biliary fistula. ${ }^{56}$ A retrospective analysis of treatment outcome in 7 of 542 patients with AHDs who received surgical treatment in our center from January 2012 to August 2020 was conducted.

A total of 542 patients received CBD surgery in the Department of General Surgery, Zhejiang University Children's Hospital from January 2012 to August 2020. Most of the patients presented with typical choledochal dilatation during surgery and underwent classic cholecystectomy with dilated biliary duct and Roux-en-Y anastomosis of the hepatic duct and jejunum. Of these 542 cases, 7 patients had anatomic variations of AHDs, and choledochojejunostomy was performed differently. Of these seven patients, two were male and five were female, with an average age of 23.5 months, ranging from 2 to 48 months. Only one case was identified by preoperative magnetic resonance cholangiopancreatography (MRCP) as having AHDs (figure 1), and the rest were found intraoperatively.
Of these seven cases, two cases were found to have choledochal cysts during prenatal examination, five cases were found to be due to abdominal pain in hospital examination, and two of them were found to be abnormal liver function with increased direct bilirubin of $61.6 \mu \mathrm{mol} / \mathrm{L}$ and $35.3 \mu \mathrm{mol} / \mathrm{L}$, alanine aminotransferase (ALT) of $119 \mathrm{U} / \mathrm{L}$ and $78 \mathrm{U} / \mathrm{L}$, and $\gamma$-glutamyltransferase of $1278 \mathrm{U} / \mathrm{L}$ and $496 \mathrm{U} / \mathrm{L}$. Routine preoperative examination included routine blood tests, $\mathrm{C}$ reactive protein level, liver function, coagulation function, orthotopic chest radiography, electrocardiogram (ECG), abdominal B-mode ultrasound and MRCP.

The patients were placed in the supine position with the head high and feet low. A $5 \mathrm{~mm} 30^{\circ}$ laparoscope was used. Four punctures for $1-25 \mathrm{~mm}$ trocars and the rest were $3 \mathrm{~mm}$ trocars were located in the left upper abdomen, right upper abdomen, navel and right lower abdomen, respectively. The anatomical separation of the extrahepatic biliary tract and treatment of the jejunal segment were basically the same as those for typical CBD, the jejuno-jejunostomy was extracorporeal, the proximal intestine and biliary branches were retained at $15-25 \mathrm{~cm}$. The difference was mainly in the hepaticojejunostomy during surgery. All the operations were performed under the 'four-hole method' laparoscopy, and there were no cases of conversion to laparotomy. The average operation time was $157 \mathrm{~min}$ (range 145-210 min) compared with $142 \mathrm{~min}$ without AHD, and intraoperative bleeding was less than $10 \mathrm{~mL}$. AHD was found in one case by preoperative magnetic resonance imaging (MRI) examination, abnormal bile duct was found in four cases when the neck of gallbladder was dissected, and AHD was found in the other two cases when bile exudation was found on the wound surface during irrigation. The 


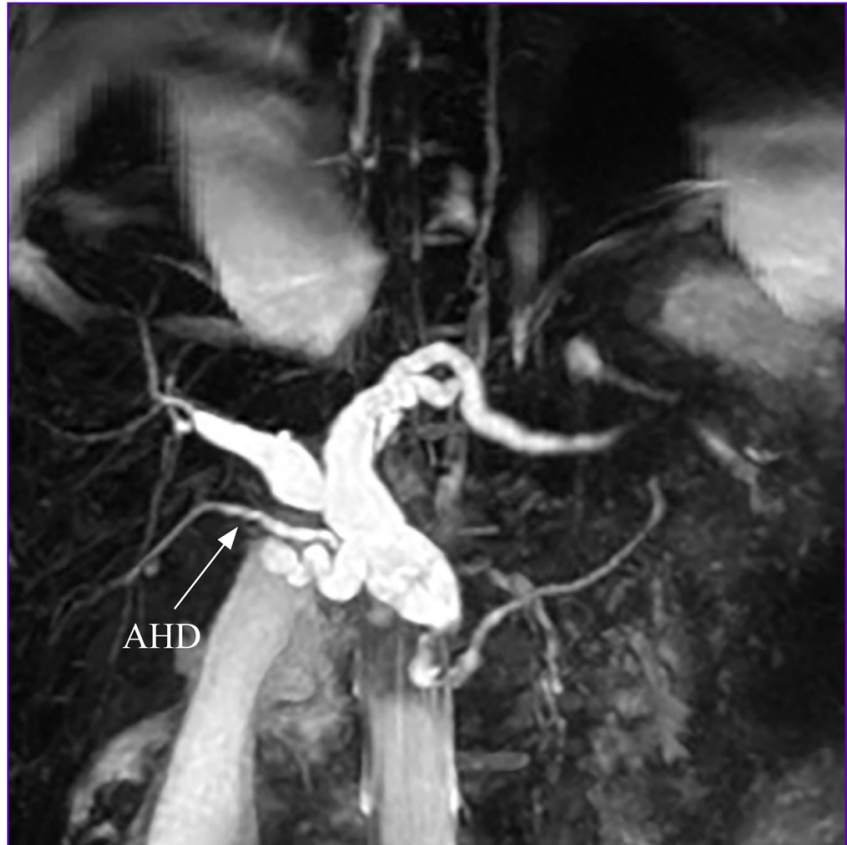

Figure 1 MRCP shows the AHD before surgery. AHD, accessory hepatic duct; MRCP, magnetic resonance cholangiopancreatography.

diameter of AHD was obtained by comparing with the tip of the laparoscopic forceps, and the diameter of the electrocoagulation hook was considered to be $1 \mathrm{~mm}$ (figure 2).

The seven cases of CBD with AHD variation were treated differently according to the size and location of the AHD and were divided into three surgical methods as shown in table 1. Method 1: after intermittent suture of the AHD and CHD, a fusiform choledochal-jejunum anastomosis was performed (three cases in total), which is shown in figure 3. Method 2: the AHD and CHD were anastomosed with the jejunum, respectively (two anastomotic stoma), in two cases (figure 4). Method 3: ligation

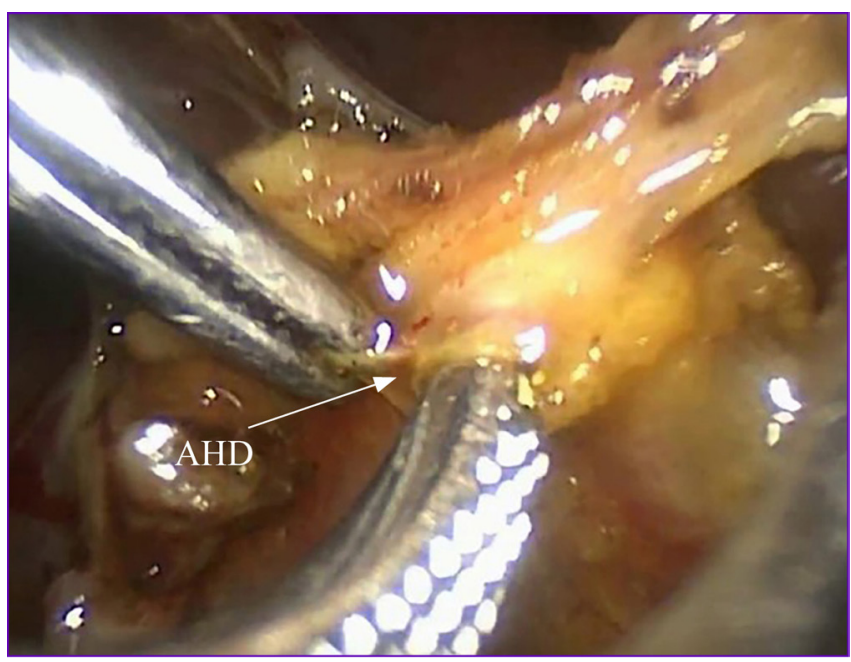

Figure 2 Measurement of AHD diameter. AHD, accessory hepatic duct. of the AHD (with diameter less than $2 \mathrm{~mm}$ ) in two cases (figure 5).

CBD is a common congenital biliary tract anomaly. The preferred radical treatment is biliary tract resection with biliary tract Roux-en-Y reconstruction. In 1995, Farello et al first reported laparoscopic surgery for CBD. ${ }^{7}$ In 2002, $\mathrm{Li}$ et al first reported laparoscopic treatment of CBD in China. ${ }^{8}$ In children with $\mathrm{CBD}$, the presence of a variant bile duct and AHD also may affect the surgical procedure. The rate of biliary tract variations is high, and the variation forms are complex and changeable. The classic normal biliary tract structure accounts for only $57.6 \%$, and the variation rate can be as high as $42.4 \% .{ }^{9}$ AHDs are variations of the biliary tract that drain the bile ducts of one lobe or segment of the liver, join into the gallbladder, common bile duct, CHD, and right hepatic duct. It is reported that the incidence of AHDs is $1.0 \%-31.4 \% .^{10}$ However, the proportion of AHDs encountered during CBD surgery is not high, and the diameters of the AHDs are usually small. Most of them cannot be clearly defined by preoperative MRCP and intraoperative angiography. In most cases, bile extravasation is often detected by careful examination during the operation. We found seven cases complicated by AHDs during surgery, and the incidence was approximately $1.3 \%$ ( 7 of 542 cases). Children's AHDs are often found to be only 1-2 $\mathrm{mm}$ in diameter, and both preoperative ultrasound and MRCP are often unable to clearly show the shape of AHDs. We do not regularly perform preoperative ERCP imaging in children with CBD, and intraoperative biliary angiography cannot clearly identify AHDs because the image is overlapped by the gallbladder or choledochal cyst. The presence of AHDs is identified by the presence of small ducts or bile leakage during surgery (figure 4). The Japanese scholar Takahashi ${ }^{5}$ summarized five CBD cases of AHDs: only one case was found during intraoperative imaging, one case was found by repeated endoscopic retrograde cholangiopancreatography (ERCP) imaging, and the rest were found by intraoperative or postoperative biliary fistula. It has been reported in the literature that more cases of AHD were found due to bile leakage during surgery or due to the postoperative complication of biliary fistula. ${ }^{611}$ In this group of seven cases, preoperative imaging with MRCP showed the presence of AHD in only one case, and in most of the children the presence of AHD before surgery could not be predicted. In four cases, small ducts were found during the operation, which were confirmed to be AHDs after careful dissection. In two cases, bile leakage was found during rinse examination of wound surface after dissection, and AHD injury was found on subsequent examination; thus, careful assessment of anatomy during the operation is particularly important. The junctions of the AHDs in these seven children were located in the gallbladder or in the neck of the gallbladder duct. During the operation, the anatomy of this site should be monitored carefully to distinguish the aberrant blood vessels from the $\mathrm{AHD}$, and it is also necessary to wash the wound surface 
Table 1 Seven cases of CBD associated with AHD

\begin{tabular}{llllll}
\hline Case & Sex & Diameter $(\mathbf{m m})$ & Surgical method & Identification & Complication \\
\hline 1 & F & 2 & 2 & During OP & - \\
2 & F & 2 & 1 & MRCP & - \\
3 & M & 1 & 3 & Bile leakage & - \\
4 & F & 2 & 1 & During OP & - \\
5 & M & 2 & 3 & During OP & - \\
6 & F & 2 & 2 & Bile leakage & - \\
7 & F & 1 & 1 & During OP & - \\
\hline
\end{tabular}

$\mathrm{AHD}$, accessory hepatic duct; $\mathrm{CBD}$, congenital biliary dilatation; F, female; $\mathrm{M}$, male; MRCP, magnetic resonance cholangiopancreatography; OP, operation.

during the operation to avoid the occurrence of postoperative bile leakage. If the diameter of the AHD is more than $2 \mathrm{~mm}$, it is feasible to separate the AHD by careful assessment of anatomy during the operation when there is no chronic inflammation of the biliary tract and local adhesions are not serious. If the AHD is too small (eg, diameter $<2 \mathrm{~mm}$ ) or if the anatomical region is not clear because of repeated infection of the cyst, it is difficult to avoid injury to the AHD. The AHD has the function of bile drainage, and anastomosis should be carried out as far as possible if injury of the AHD is found during the operation. Wen Zhe proposed that biliary tract reconstruction is recommended for AHD over $2 \mathrm{~mm} .{ }^{12}$ In our center, three of seven cases whose AHDs (approximately $2 \mathrm{~mm}$ in diameter) were around the CHD were anastomosed to the branch of the jejunal biliary tract with a spindle shape after intermittent suture of the AHD and the lateral wall of the CHD to ensure bile drainage. Two of seven cases whose AHD diameter was $2 \mathrm{~mm}$ but also a certain distance from the CHD could not be combined together. Therefore, two choledochojejunostomies were performed separately. In the remaining two cases whose
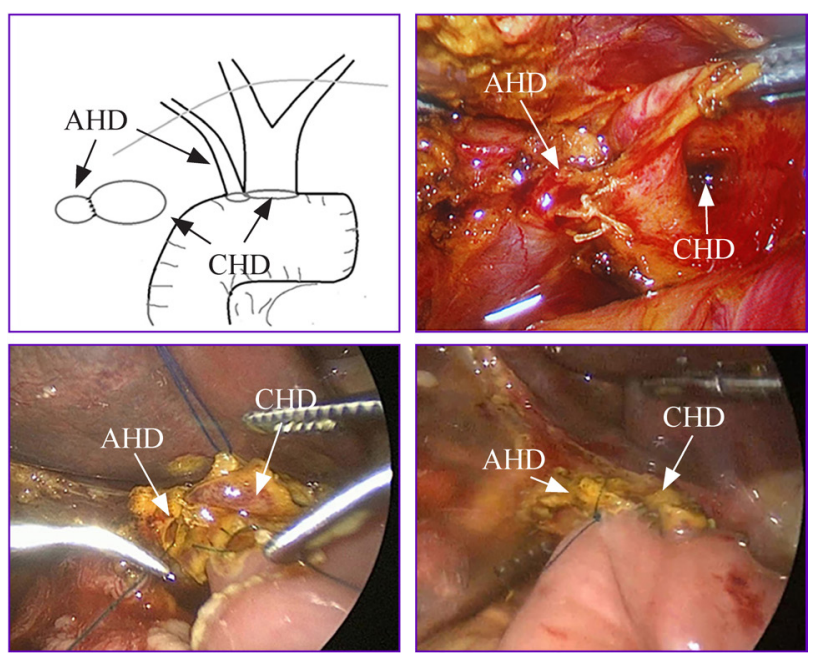

Figure 3 Anastomosed to the branch of the jejunal biliary tract with a spindle shape after intermittent suture of the accessory hepatic duct (AHD) and the lateral wall of the common hepatic duct (CHD).
AHD diameter was less than $2 \mathrm{~mm}$, which was the nonanastomotic type, simple ligation was performed. After the above treatment, all patients were followed up for 2-23 months. None of the patients had abnormal liver function or elevated bilirubin, and B-mode ultrasound examination showed no dilatation of the biliary tract inside and outside the liver. It is safe and feasible to ligate the AHD with a diameter less than $2 \mathrm{~mm}$. Although anastomosis of the AHD with a diameter greater than $2 \mathrm{~mm}$ is difficult and requires an experienced surgeon, postoperative complications, such as increased bilirubin, biliary fistula and biliary stricture are rare; thus, anastomosis is recommended. If the AHD is close to the hepatic duct, a spindle anastomosis after tenseless combination can reduce the difficulty of anastomosis, which is a safe and effective surgical method.

Postoperative recovery was uneventful. No postoperative bleeding, biliary fistula, intestinal fistula, biliary obstruction or ileus complications occurred during hospitalization. The average postoperative hospital stay was 10 days and ranged from 8 to 14 days. For anastomotic stenosis occurring months to years after surgery, long-term follow-up is maintained. The postoperative follow-up period ranged from 2 months to 23 months, and the follow-up indices measured included serum bilirubin, ALT, routine blood tests, C reactive protein and ultrasonography. For the two cases with abnormal liver function before surgery, with direct bilirubin of $61.6 \mu \mathrm{mol} / \mathrm{L}$, ALT of $119 \mathrm{U} / \mathrm{L}$ and $\gamma$-glutamyltransferase of $1278 \mathrm{U} / \mathrm{L}$, these values returned to normal 19 days after surgery in case 1.
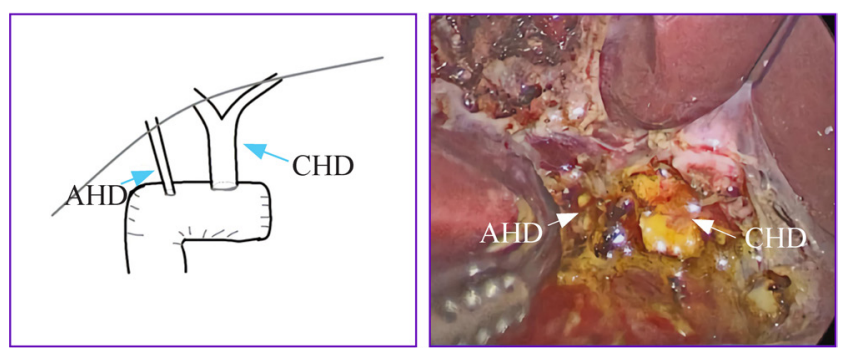

Figure $4 \mathrm{AHD}$ and $\mathrm{CHD}$ were anastomosed with the jejunum, respectively (two anastomotic stomas). AHD, accessory hepatic duct; CHD, common hepatic duct. 


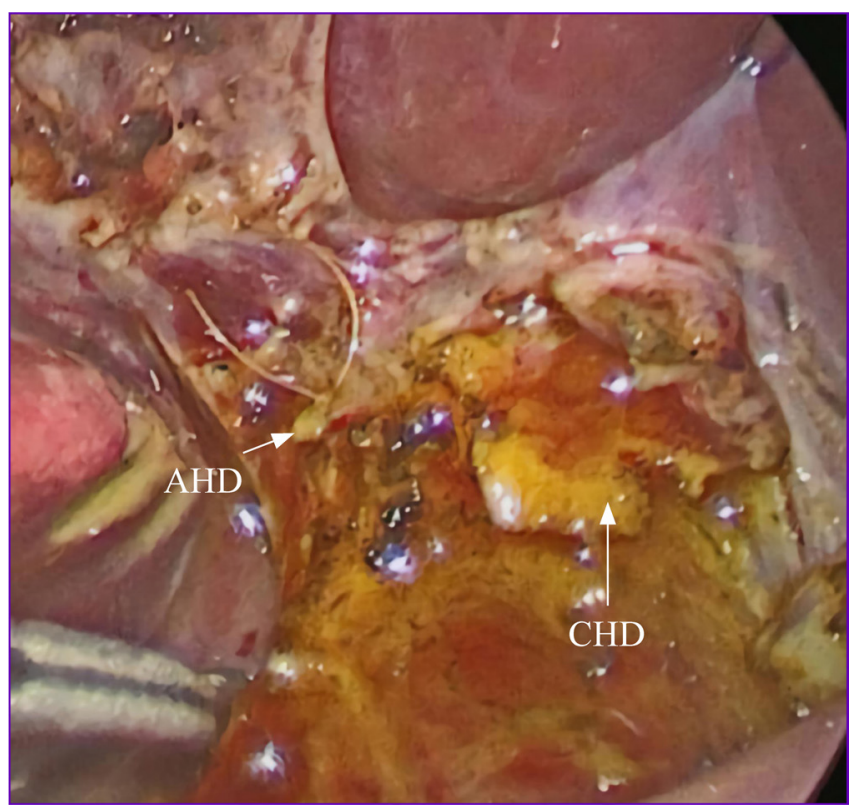

Figure 5 Ligation of the AHD. AHD, accessory hepatic duct; $\mathrm{CHD}$, common hepatic duct.

Direct bilirubin of $35.3 \mu \mathrm{mol} / \mathrm{L}$, ALT of $78 \mathrm{U} / \mathrm{L}$ and $\gamma$-glutamyltransferase of $496 \mathrm{U} / \mathrm{L}$ returned to normal 13 days after surgery in case 2 . The seven patients with CBD and AHDs did not have an abnormal increase in bilirubin, and biliary ultrasonography showed no obvious dilatation of the biliary tract inside and outside the liver 2-23 months after surgery.

In summary, biliary ducts of patients with CBD have varied dilated locations and forms and are often associated with variable biliary ducts and blood vessels. For the treatment of AHD, it is necessary to dissect and identify the abnormal vessels and AHDs carefully during the operation, and it is also necessary to wash the wound after the dissection. If the diameter of the AHD is larger than $2 \mathrm{~mm}$, choledochojejunostomy should be performed. The surgical methods included two independent choledochojejunostomies when the AHD is far from the CHD, and one spindle anastomosis when the AHD and CHD are close.

\section{Twitter Zhigang Gao @gzg2k}

Contributors YZ contributed to investigation, data curation and writing (original draft). ZG was responsible for data curation, project administration, resources, supervision, visualization and writing (review and editing). JY carried out data curation, supervision, validation, visualization and writing (review and editing). DC and $\mathrm{CQ}$ performed data curation, project administration, resources and supervision.
Funding This work was supported by Medical Health Science and Technology Project of Zhejiang Province (grant number 2017KY434).

Competing interests None declared.

Patient consent for publication Not required.

Ethics approval This study is a retrospective study, which does not involve patients' privacy and does not infringe patients' interests and health. The study was conducted in accordance with the principles of Declaration of Helsinki, and the study protocol was approved by the ethics committee of Zhejiang University School of Medicine Children's Hospital.

Provenance and peer review Not commissioned; externally peer reviewed.

Data availability statement The data that support the findings of this study are available from the corresponding author upon reasonable request.

Open access This is an open access article distributed in accordance with the Creative Commons Attribution Non Commercial (CC BY-NC 4.0) license, which permits others to distribute, remix, adapt, build upon this work non-commercially, and license their derivative works on different terms, provided the original work is properly cited, appropriate credit is given, any changes made indicated, and the use is non-commercial. See: http://creativecommons.org/licenses/by-nc/4.0/.

\section{ORCID iD}

Yuebin Zhang http://orcid.org/0000-0001-9295-9398

\section{REFERENCES}

1 Todani T, Watanabe Y, Narusue M, et al. Congenital bile duct cysts: classification, operative procedures, and review of thirty-seven cases including cancer arising from choledochal cyst. Am J Surg 1977;134:263-9.

2 Soares KC, Arnaoutakis DJ, Kamel I, et al. Choledochal cysts: presentation, clinical differentiation, and management. J Am Coll Surg 2014;219:1167-80.

3 Ronnekleiv-Kelly SM, Soares KC, Ejaz A, et al. Management of choledochal cysts. Curr Opin Gastroenterol 2016;32:225-31.

4 Section of Laparoscopic\&Endoscopic Surgery, Branch of Pediatric Surgery,Chinese Medical Association. Guideline for laparoscopic hepatojejunostomy for choledochal cyst in children(2017). Chin J Pediatr Surg 2017;38:485-94. http://rs.yiigle.com/CN421158201707/ 1008537.htm

5 Takahashi T, Shimotakahara A, Takahashi T, et al. Choledochal cyst associated with an accessory hepatic duct identified by intraoperative endoscopy: case report and literature review. Pediatr Surg Int 2008;24:1079-82.

6 Narasimhan KL, Chowdhary SK, Rao KL. Management of accessory hepatic ducts in choledochal cysts. J Pediatr Surg 2001;36:1092-3.

7 Farello GA, Cerofolini A, Rebonato M, et al. Congenital choledochal cyst: video-guided laparoscopic treatment. Surg Laparosc Endosc 1995;5:354-8.

8 Li L, Feng W, Jing-Bo F, et al. Laparoscopic-assisted total cyst excision of choledochal cyst and Roux-en-Y hepatoenterostomy. $J$ Pediatr Surg 2004;39:1663-6.

9 Puente SG, Bannura GC. Radiological anatomy of the biliary tract: variations and congenital abnormalities. World J Surg 1983;7:271-6.

10 Kimura $\mathrm{H}$, Ishida $\mathrm{H}$, Konno $\mathrm{K}$, et al. Accessory hepatic duct: sonographic findings. Abdom Imaging 2002;27:329-32.

11 HW X, Zhao Z, Duan WQ. Double Y-shaped anastomotic mode in the treatment of congenital bile duct dilatation plus aberrant bile ducts. Chin J Pediatr Surg 2015;36:690-3.

12 Wen Zhe L. Diagnosis and management of congenital choledochal cyst plus accessory hepatic duct. Chin J Pediatr Surg 2019;18:533-5. 